\title{
The Numerical Synthesis of a Radar Signal Having Required Autocorrelation Function
}

\author{
Cz. Leśnik, A. Kawalec and M. Szugajew* \\ Institute of Radio-Electronics, Military University of Technology, Warsaw, Poland
}

\begin{abstract}
This paper presents the problem of synthesis of signals modulated in frequency with autocorrelation function that implements an optimal approximation to a given autocorrelation function. After theoretical introduction an algorithm of frequency modulated signal synthesis is presented. Simulation results made in Matlab are presented in the last section.
\end{abstract}

PACS numbers: 41.20.Jb, 03.50.De

\section{Introduction}

Nowadays radars are becoming increasingly common and their applications are growing beyond closely military applications. Their structure is also evolving violently. Most common radar waveform is the linear FM (LFM) signal. Its utility is that it is fairly readily generated by a variety of technologies, and is easily processed by a variety of techniques that ultimately implement a matched filter, or nearly so. However, since a LFM waveform has nearly rectangular power spectrum density (PSD), its autocorrelation function exhibits a $\frac{\sin x}{x}$ function shape, with its attendant problematic side lobe structure [1]. This enables a worldwide search of an algorithm that would enable synthesis of signals that would have desired properties. One of the most promising group of signals are signals modulated in frequency with nonlinear frequency modulation function (NLFM). Such a frequency modulation function can shape the autocorrelation function (by shaping power spectrum) to have much lower side lobes than LFM signal would have. As a result there is no need to apply window functions to reduce autocorrelation function side lobe levels. There are two main constraints imposed on the NLFM signal:

- it must be easy to produce,

- it must be easy to process at the receiver.

With the progress of modern technology these points are becoming easier to meet, especially thanks to the progress in high speed analog to digital converters and progress in field programmable gate arrays (FPGA). This suggests that generation of more complex signals is becoming possible.

Why is the autocorrelation function so important in radar applications? Autocorrelation function is a reaction of the receiving system (optimum in scope of signal

\footnotetext{
* corresponding author; e-mail:

marcin.szugajew@wel. wat.edu.pl
}

to noise ratio minimization) to the signal reflected from the target [2-4]. In case of distance measurement it describes accuracy and resolution of such measurements. Matched filter is a solution to the problem of optimum filtration in scope of signal to noise ratio minimalization. Signal on the exit of such filter is proportional to the autocorrelation function i.e.

$$
s(t)=A R(\tau)
$$

where $A$ - proportionality coefficient, $R(\tau)$ - autocorrelation function.

One can see that selection of the signal is a very important issue. One would want to have a signal of whom the autocorrelation function $R(\tau)$ would be "similar" to certain "perfect" $R_{\mathrm{opt}}(\tau)$ in sense of criterion that would provide desirable property or properties. In signal synthesis there are two possibilities for above mentioned criterion $f$ : min-max or square criterion of similarity. In this paper we will be using the latter one

$$
f=\int_{-\infty}^{\infty}\left[\left|R_{\mathrm{opt}}(\tau)\right|-|R(\tau)|\right]^{2} \mathrm{~d} \tau .
$$

In addition we assume that the energy spectrum $G(\omega)$ of the signal $s(t)$,

$$
G(\omega)=0 \text { for }|\omega|>\Omega
$$

is non-zero in finite range of frequencies.

Autocorrelation function does not determine signal $s(t)$ in explicit way, it only determines signals amplitude spectrum

$$
\begin{aligned}
& R(\tau)=\frac{1}{2 \pi} \int_{-\infty}^{\infty}|S(\omega)|^{2} \mathrm{e}^{\mathrm{j} \omega \tau} \mathrm{d} \omega, \\
& |S(\omega)|^{2}=\int_{-\infty}^{\infty} R(\tau) \mathrm{e}^{-\mathrm{j} \omega \tau} \mathrm{d} \tau,
\end{aligned}
$$

where $|S(\omega)|$ - amplitude spectrum.

From the following one can see that any signal with given amplitude spectrum and random phase spectrum can have the desired autocorrelation function.

In the next section of this paper the problem of synthesis of signals modulated in frequency with autocorrelation function that implements an optimal approximation to a 
given autocorrelation function will be presented. In the last section simulation results will be shown.

\section{Signal synthesis algorithm}

In this part of the article essential parts of the signal synthesis algorithm are presented.

The synthesis of optimum signal $x_{\mathrm{opt}}$ is equivalent to determination of shortest distance $d(x, y)$, in sense of square criterion, between the $X$ and $Y$ sets, i.e.

$$
d_{\min }=\min _{x \in X} d(x, y) \text {. }
$$

Square criterion specifies a rule according to which to each pair of functions $x$ and $y$ distance $d(x, y)$ is assigned to. The distance $d(x, y)$ is often called a function space metric and should not be interpreted as the geometric distance [5, 6] (Fig. 1).

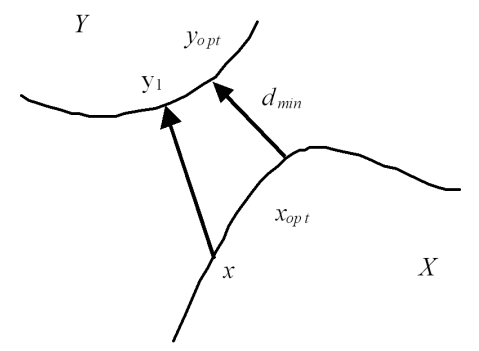

Fig. 1. Geometrical interpretation of signal synthesis task.

There are several ways to solve the problem of signal synthesis, i.e., finding a signal $x$ with lowest value of distance $d_{\mathrm{min}}$. In the following part of the article one of them will be discussed.

In the first step one chooses facultative signal $x \in X$ and determines the best approximation on $Y$ set of signals. The quality of approximation is characterized by a distance expressed by the following:

$$
d(x, Y)=\min _{y \in Y} d(x, y)=\|x-y\|,
$$

where $\|\cdot\|-$ norm of the signal.

This distance corresponds to a specific signal $y_{1}$ (Fig. 1). If the signal does not have the desired properties, the signal $x$ is changed (moving inside set $X$ ), and the distance $d(x, y)$ is determined again. Successive distances values should form a decreasing sequence

$$
d_{1}>d_{2}>d_{3}>\ldots
$$

This operation is repeated until the minimum of (6) is achieved. It should be noted that $X$ is a set of signals that are characterized by an autocorrelation function $R(\tau)$ and $Y$ is the set of signals that possesses the desired autocorrelation function.

Based on the above method a numerical procedure of designing the time-frequency structure of signals modulated in frequency with desired autocorrelation function was developed. In the following parts of this article essential elements of this method are presented.
The task of signal synthesis is to synthesize the frequency modulation function $\omega_{\mathrm{c}}(t)$ that realizes the best approximation to the desired signal $y(t)$. Suppose that elements of set of possible signals $X$ are given as

$$
x(t)=B(t) \mathrm{e}^{\mathrm{j} \varphi(t)} \quad \text { for } \quad|t| \leq \frac{T}{2},
$$

where $T$ - duration of the pulse, $B(t)$ - signal envelope, $\varphi(t)$ — phase modulation function.

Spectrum of signal $x(t)$ :

$\tilde{X}(\omega)=b_{x}(\omega) \mathrm{e}^{-\mathrm{j} \beta_{x}(\omega)}$,

where $b_{x}(\omega)$ - amplitude spectrum, $\beta_{x}(\omega)$ - phase spectrum.

In the process of synthesis, it is assumed that the envelope of signal $(B(t))$ is set, and the function $\varphi(t)$ is arbitrary. Frequency modulation function is expressed as follows:

$$
\omega_{\mathrm{c}}(t)=\frac{\mathrm{d} \varphi(t)}{\mathrm{d} t} .
$$

Elements of $Y$ have the following form:

$$
y(t)=A(t) \mathrm{e}^{\mathrm{j} \Phi(t)},
$$

and their spectra

$$
\tilde{y}(\omega)=a(\omega) \mathrm{e}^{\mathrm{j} \alpha(\omega)},
$$

where $A(t)$ - envelope of signal $y(t), \Phi(t)$ — phase function of signal $y(t), \alpha(\omega)$ - phase spectrum of signal $\tilde{y}(\omega)$, $a(\omega)$ - amplitude spectrum of signal $\tilde{y}(\omega)$.

Signals $x(t) \in X$ differ only in the form of phase modulation function $\varphi(t)$, with given envelope $a(\omega)$. With given functions $a(\omega)$ and $B(t)$ one synthesizes phase modulation function $\varphi(t)$ (or $\beta_{x}(\omega)$ ), which minimizes the distance between $X$ and $Y$ sets [2]. This means that one must determine the similarity coefficient

$$
C(x, Y)=\frac{1}{2 \pi} \int_{-\infty}^{\infty} a(\omega) b_{x}(\omega) \mathrm{d} \omega=\max ,
$$

where

$$
b_{x}(\omega)=|\tilde{x}(\omega)|=\left|\int B(t) \mathrm{e}^{\mathrm{j}[\varphi(t)-\omega t]} \mathrm{d} t\right| .
$$

Optimal frequency modulation function, which maximizes the above similarity coefficient shall be determined on the basis of the differential equation

$$
B^{2}(t) \mathrm{d} t=\frac{1}{2 \pi} a^{2}\left(\omega_{\mathrm{c}}\right) \mathrm{d} \omega_{\mathrm{c}} .
$$

After determining the frequency modulation function $\omega_{\mathrm{c}}(t)$ from the above equation, in the next step the phase modulation function is determined as

$$
\varphi(t)=\int \omega_{\mathrm{c}}(t) \mathrm{d} t .
$$

In order to determine the phase spectrum of signal $x(t)$ one must resolve the integral

$$
\tilde{X}(\omega)=\int_{-T / 2}^{T / 2} B(\omega) \mathrm{e}^{\mathrm{j}[\varphi(t)-\omega t]} \mathrm{d} t .
$$

Using the method of stationary phase [2] one obtains a solution of the form

$$
\beta_{x}(\omega)=-\left[\varphi(t)-\omega t_{0} \pm \frac{\pi}{4}\right],
$$

where $t_{0}$ - point of stationary phase. 


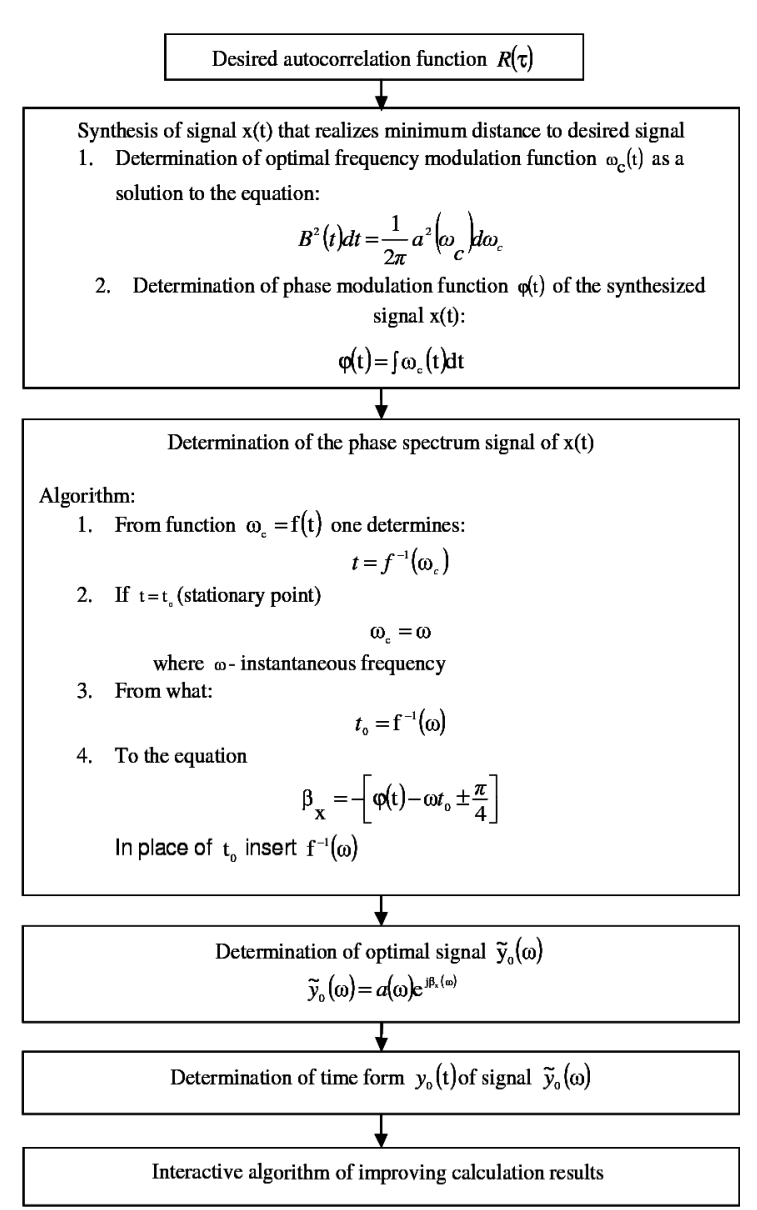

Fig. 2. Signal synthesis algorithm.

In the next step, from the above equation phase spectrum $\beta_{x}(\omega)$ should be determined, which is then mapped to a specified amplitude spectrum $a(\omega)$, in order to obtain signal $y(t)$ closest to the signal $x(t)$ :

$$
\tilde{y}_{0}(\omega)=a(\omega) \mathrm{e}^{\mathrm{j} \beta_{x}(\omega)} .
$$

The determined spectrum $\beta_{x}(\omega)$ is both phase spectrum of signal $x_{\mathrm{opt}}(t)$ and signal $y_{0}(t)$. Signal $y_{0}(t)$ is obtained by taking the inverse Fourier transform from signal $\tilde{y}_{0}(\omega)$. At this stage, namely after determination of signal $x_{\mathrm{opt}}(t)$, signal synthesis could be ended, but the results will be affected by the error introduced by the used stationary phase method. In order to reduce them an iterative method was constructed.

The essential steps of signal synthesis algorithm are presented in graphical manner in Fig. 2.

\section{Simulation results}

Algorithm presented in the previous chapter was implemented using Matlab. Firstly, correctness of operation was checked by synthesis of LFM signal with rectangular amplitude spectrum, given by

$$
a(\omega)=\left\{\begin{aligned}
1 / \sqrt{\pi / \omega} & \text { for } \quad-\Omega \leq \omega \leq \Omega, \\
0 & \text { for }|\omega|>\Omega .
\end{aligned}\right.
$$

Secondly, NLFM signal was synthesized. Amplitude spectrum of this signal is given by

$$
\begin{gathered}
a(\omega)=\sqrt{\frac{\pi}{\Omega}\left(1+g \cos \left(\pi \frac{\omega}{\Omega}\right)\right)} \\
\text { for }-\Omega<\omega<\Omega .
\end{gathered}
$$

Envelope of both synthesized signals was rectangular

$$
B(t)=\left\{\begin{array}{c}
1 / \sqrt{T} \quad \text { for } \quad-T / 2 \leq t \leq T / 2, \\
0 \text { for } \quad|t>T / 2| .
\end{array}\right.
$$

Frequency modulation functions for the both LFM and NLFM signals (the solution of the differential Eq. (14)), are presented graphically in Figs. 3 and 4.

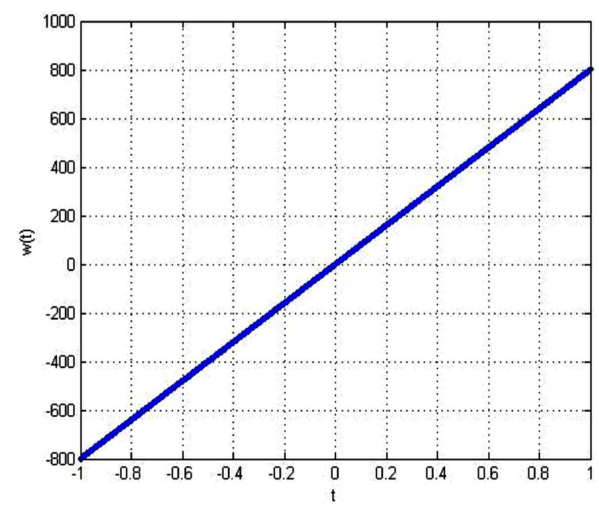

Fig. 3. Frequency modulation function, LFM signal.

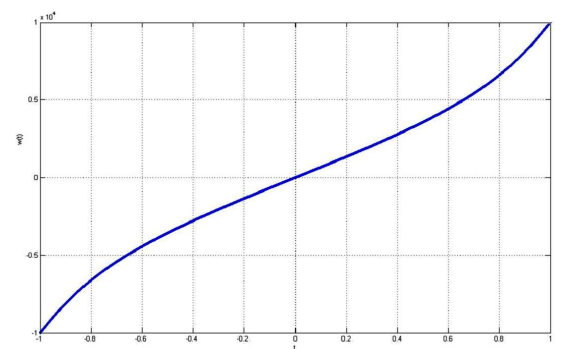

Fig. 4. Frequency modulation function, NLFM signal $(g=0.5)$.

Amplitude spectra of synthesized signals are presented graphically in Figs. 5 and 6.

In the second experiment parameter $g$ from Eq. (20) was changed from value zero to 0.995 . With this parameter one can adjust the autocorrelation function by adjusting "nonlinearity" of frequency modulation function. The change in the frequency modulation function can be seen in Figs. 7 and 8 where the parameter $g$ was changed from value 0.2 to 0.995 .

As mentioned in the previous paragraph additional algorithm for reducing errors introduced by the station- 


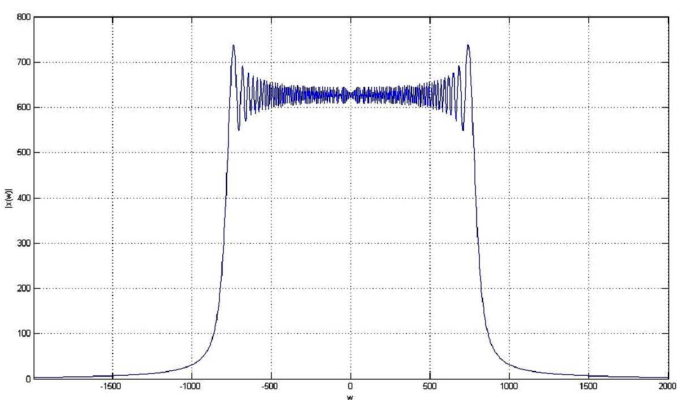

Fig. 5. Amplitude spectrum of the synthesized signal (LFM).

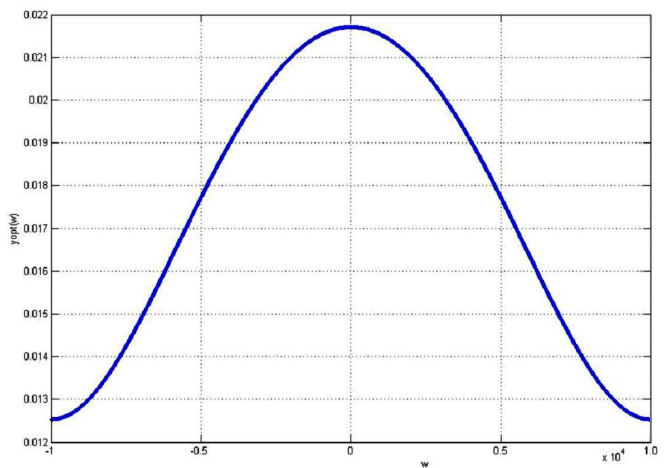

Fig. 6. Amplitude spectrum of the synthesized signal $(g=0.5)$.

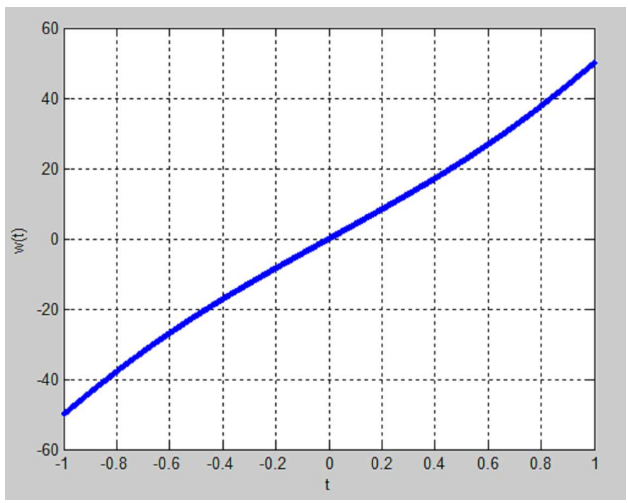

Fig. 7. Frequency modulation function, NLFM signal $(g=0.2)$.

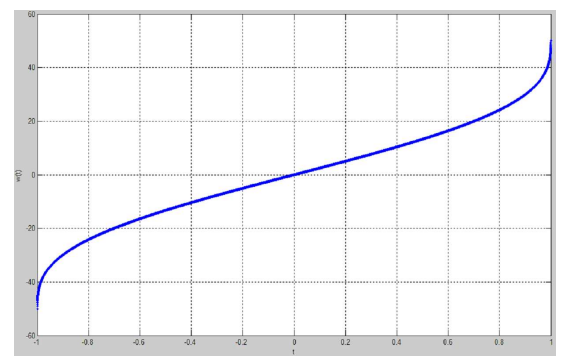

Fig. 8. Frequency modulation function, NLFM signal $(g=0.995)$. ary phase method used for solving integral (16) was constructed. Result of our work can be seen in Figs. 9, 10 and 11. In Fig. 9 autocorelation function of zero approximation was plotted. In Fig. 10 the same autocorrelation function was plotted, but after going through one iteration of second algorithm.

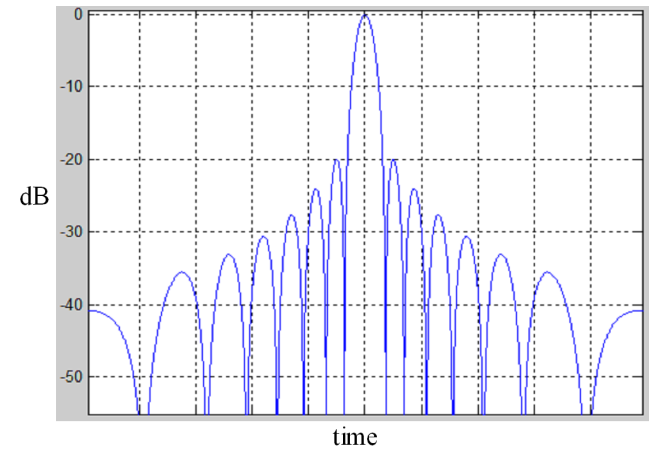

Fig. 9. Autocorelation function of the NLFM signal zero approximation $(g=0.2)$.

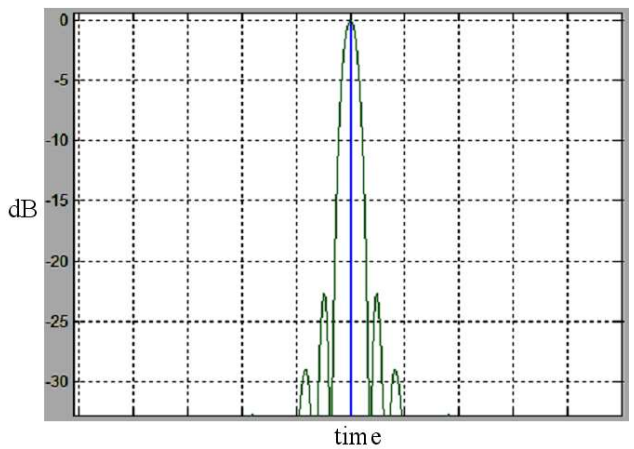

Fig. 10. Autocorelation function of the NLFM signal after first iteration $(g=0.2)$.

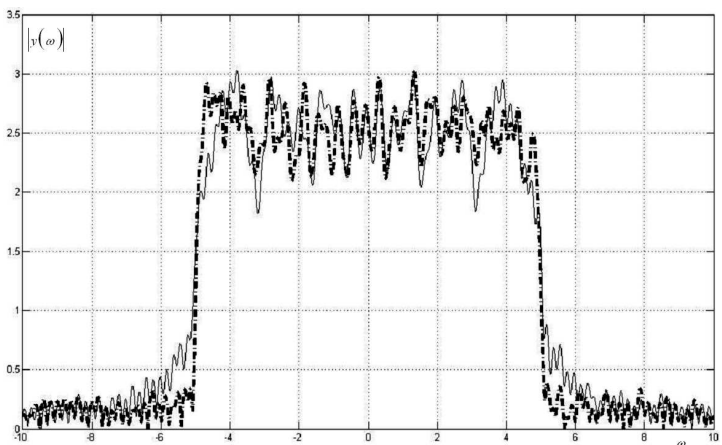

Fig. 11. Amplitude spectrum of the synthesized LFM signal zero approximation (solid) and 30th iteration (dash).

As one can see, the first side lobe of autocorelation function was reduced by $3 \mathrm{~dB}$. The amount of decrease 
is small when the product bandwidth times pulse duration is big and gets larger when this product is small. This is because the stationary phase method is less acurate when the product bandwidth times pulse duration is small. The second reason of such behavior is that when the change in power spectrum is big (it can be especially seen in the LFM signal whose ideal power spectrum is rectangular) used equations are subject to error. When the change in PSD is smooth the error is small.

In Fig. 11 amplitude spectra of synthesized LFM signals are plotted. Curve one is the zero approximation spectrum and curve two is after thirty iterations of second algorithm. As one can see, amplitude spectrum is near to the optimal impossible to meet rectangular amplitude spectrum.

In Fig. 12 one can see the change in autocorrelation functions first side lobe level in function of $g$ parameter.

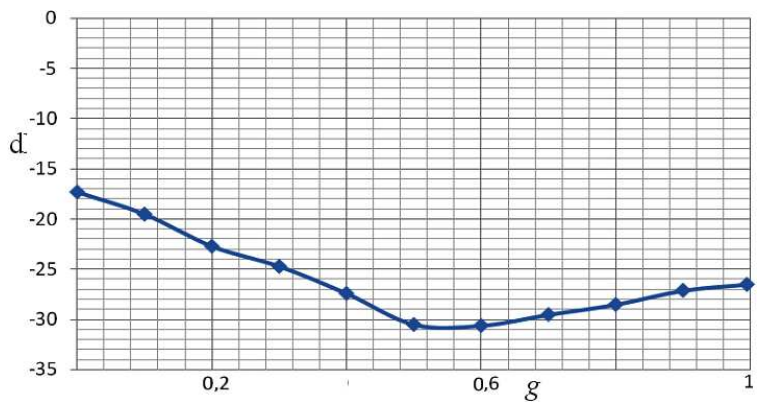

Fig. 12. Change of autocorrelation functions first side lobe level with the change of parameter $g$.

From this figure it can be seen that side lobe level decreases as $g$ parameter is increased. This decrease ends when $g$ parameter reaches 0.5 value after which side lobe level starts to increase. This rises a separate problem of selection of the optimal frequency modulation function (power spectrum) in order to achieve the desired side lobe level of the autocorrelation function with determined width of the main lobe. This problem is being addressed.

\section{Conclusion}

This article discusses key aspects of signal synthesis needed for the selection of signals with desired autocorrelation function, for example in radar technology. Results of previous studies as theoretical and numerical results confirm the usefulness of the method discussed in the article from the standpoint of signal optimization. This method is useful for cases where the desired autocorrelation function and the subsequent results of the calculations cannot be represented in the strict analytical form. Nevertheless presented algorithm allows numerical methods to find the optimal solution, having at the entrance only a discrete set of signal points. The next step is to find a power spectrum that would give a possibility of selection of width of main lobe of the autocorrelation function with lowest possible side lobe levels. In general, this power spectrum can be impossible to achieve. Signal closest to such a power spectrum can be synthesized by presented algorithms.

\section{Acknowledgments}

This work was supported by the National Centre for Research and Development for the years 2007-2010 under Commissioned Research Project PBZ-MNiSW-DBO$-04 / \mathrm{I} / 2007$

\section{References}

[1] A.W. Doerry, Generating Nonlinear FM Chirp Waveforms for Radar, Sandia Report, Sandia National Laboratories, 2003.

[2] C.E. Cook, M. Bernfeld, Radar Signals: An Introduction to Theory and Application, Artech House, Norwood, MA 1993.

[3] A. Kawalec, C. Ziołkowski, C. Leśnik, J. Pietrasinski, Acta Phys. Pol. A 116, 335 (2009).

[4] P. Kaniewski, Acta Phys. Pol. A 114, A-131 (2008).

[5] D.E. Vakman, R.M. Sedletzkiy, The Issues of Radar Signal Synthesis, Sovietskoye Radio, Moscow 1973.

[6] C. Leśnik, Acta Phys. Pol. A 116, 351 (2009). 